\title{
Qualidade De VIDA DE CUIDAdORES DE PESSOAS COM NECESSIDADES ESPECIAIS ${ }^{1}$ \\ QUALITY OF LIFE OF CAREGIVERS OF PEOPLE WITH SPECIAL NEEDS
}

\author{
Lígia Maria Presumido BRACCIALLI ${ }^{2}$ \\ Priscilla dos Santos BAGAGI ${ }^{3}$ \\ Andréia Naomi SANKAKO ${ }^{4}$ \\ Rita de Cássia Tibério ARAÚJO 5
}

\begin{abstract}
RESUMO: a qualidade de vida de cuidadores é algo que preocupa, pois afeta diretamente a qualidade de vida do indivíduo dependente desses cuidados. Este estudo teve como objetivo analisar e comparar a qualidade de vida em saúde de cuidadores de pessoas com necessidades especiais em atendimento em uma instituição de reabilitação. Participaram 90 cuidadores principais de pessoas com necessidades especiais em atendimento em uma instituição de reabilitação. Foram utilizados para a coleta de dados dois instrumentos: um questionário para verificar o perfil do cuidador e da pessoa com necessidades especiais e o questionário de medida de qualidade de vida WHOQOL-Bref. Os resultados demonstraram que houve significância, apenas, entre a qualidade de vida do cuidador no domínio físico e a idade da pessoa com necessidade especial, indicando que quanto mais velha a pessoa com necessidades especiais, mais difícil e penoso é o ato de cuidar. Os resultados podem indicar formas de orientação à família de pessoas com deficiência.
\end{abstract}

PALAVRAS-CHAVE: Educação Especial. Cuidador. Qualidade de Vida. Pessoas com Necessidades Especiais. Família.

\begin{abstract}
The quality of life of caregivers is a concern because it directly affects the quality of life of individuals dependent on such care. This study aimed to analyze and compare the quality of life in health of caregivers of people with special needs who attend a rehabilitation facility. Ninety caregivers of people with special needs who attend a rehabilitation facility participated in this study. For data collection two instruments were used: a questionnaire to determine the profile of the caregiver and the person with special needs and the WHOQOL-Bref questionnaire to measure quality of life. The results were significant only between the caregiver's quality of life in the physical domain and age of the person with special needs, indicating that the older a person with special needs, the more difficult and arduous is the act of caring. The result can indicate guidelines for caring for families of people with disabilities.
\end{abstract}

KEYWORDS: Special Education. Caregivers. Quality of life. Persons with special needs. Family.

\footnotetext{
${ }^{1}$ Agradecemos ao CNPq pelo auxílio financeiro por meio de bolsa PIBIC.

${ }^{2}$ Livre - docente, docente do Departamento de Educação Especial da Faculdade de Filosofia e Ciências, Unesp Univ Estadual Paulista, Marília. bracci@marilia.unesp.br

${ }^{3}$ Mestre em Educação pela Faculdade de Filosofia e Ciências, Unesp - Univ Estadual Paulista, Marília e Docente da ACEG - Associação Cultural e Educacional de Garça. pbagagi@hotmail.com

${ }^{4}$ Mestre em Educação e Doutoranda em Educação da Faculdade de Filosofia e Ciências, Unesp - Univ Estadual Paulista, Marília. asankako@yahoo.com.br

${ }^{5}$ Doutora em Educação e docente do Departamento de Educação Especial da Faculdade de Filosofia e Ciências, Unesp - Univ Estadual Paulista, Marília. ritac@marilia.unesp.br
} 


\section{INTRODUÇÃo}

O grupo de pessoas com necessidades especiais é bastante heterogêneo e as variadas condições de saúde dessa população demandam intervenções que se situam nos limites entre as áreas da saúde e a da educação.

As necessidades especiais designam necessidades específicas de grupos populacionais também específicos, como por exemplo, o grupo de pessoas idosas, o de pessoas com afecções crônicas, o de pessoas em situação de vulnerabilidade social.

As pessoas com necessidades especiais, geralmente, necessitam de auxílio para a realização das atividades cotidianas, e, dessa forma, essa necessidade especial se estende para seus familiares cuidadores cuja qualidade de vida pode estar ameaçada pelas circunstâncias de cuidados frequentes dispensados àqueles. Portanto, a especificidade da necessidade especial do cuidador está diretamente relacionada à qualidade de vida no âmbito da saúde geral. E diante de situações de risco de malefício à sensação de bem-estar, as abordagens educativas ocupam lugar de destaque nos programas de intervenção.

As intervenções em saúde com foco na educação podem ser entendidas sob parâmetros ocupacionais, isto é, compreendendo-se a educação como área ocupacional da vida cotidiana. A educação enquanto área ocupacional compreende a participação da pessoa na educação formal, a exploração das necessidades ou interesses educacionais pessoais informais e a participação pessoal informal na educação, esta última significando a participação em aulas, programas e atividades que forneçam instrução/treinamento em áreas identificadas de interesse (AMERICAN OCCUPATIONAL THERAPY ASSOCIATION, 2010).

A investigação de qualidade de vida do cuidador familiar de pessoas com necessidades especiais tem repercussão na exploração das necessidades ou interesses educacionais pessoais informais, e, nessa medida, pode constituir um desdobramento de temáticas da área de Educação Especial.

A função de cuidar de pessoas com necessidades especiais comumente é exercida por um membro da família (HINRICHSEN; NIEDEREHE, 1994) que assume a função de cuidador principal. O cuidador principal é aquele que tem total ou a maior responsabilidade pelos cuidados prestados à pessoa dependente no domicílio, dedicando a maior parte do seu tempo, medido em número de horas ao dia, ao cuidado do enfermo (DWYER; LEE; JANKOWSKY, 1994).

Wilson (1989) definiu cuidador principal como aquele indivíduo procedente do sistema de apoio informal do indivíduo cuidado, seja familiar ou amigo, e que tem as seguintes características: 1) assume as principais tarefas de cuidado, com as responsabilidades que o cerca; 2) é percebido pelos demais membros da família como responsável por assumir os cuidados com o enfermo; 3) não é remunerado economicamente pelas tarefas de cuidado; 4) exerce estas atividades no mínimo seis semanas a cada três meses. 
Em geral, a decisão de assumir os cuidados é consciente, e os estudos revelam que, embora a designação do cuidador seja informal e decorrente de uma dinâmica, o processo parece obedecer a certas regras refletidas em quatro fatores: 1) parentesco, com frequência maior para os cônjuges, antecedendo sempre a presença de algum filho; 2) gênero, com predominância da mulher; 3) proximidade física, considerando quem vive com a pessoa que requer cuidados; 4) e proximidade afetiva, destacando a relação conjugal e a relação entre pais e filhos (MENDES, 1995). Outros estudos mostram que o cuidador, geralmente, é representado por uma figura feminina, ou seja, a mãe, esposa ou filha (ROIG; ABENGÓZAR; SERRA, 1998; GARRIDO; ALMEIDA, 1999; BOVER, 2004).

Como o cuidador principal é a pessoa que despende um tempo maior com os cuidados do individuo com necessidades especiais, ela pode estar exposta a uma série de consequências devido a esse fato. Assim, pode vir a ter desgastes físicos, psicológicos ou emocionais devido à sobrecarga a que está submetido.

De acordo com Westphal et al. (2005, p.73)

A sobrecarga do cuidador pode ser vista como um conceito multidimensional que abrange a esfera biopsicossocial e resulta da busca de um equilíbrio entre as variáveis: tempo disponível para o cuidado, recursos financeiros, condições psicológicas, físicas e sociais, atribuições e distribuição de papéis.

O cuidador principal por passar um tempo muito elevado atendendo às necessidades do indivíduo dependente, pode sofrer um estresse social, e tem como consequência o afastamento, muitas vezes, da sua própria família, dos amigos e uma limitação no seu convívio social (GONÇALVES, 2002).

Autores têm descritos alguns problemas enfrentados pelos cuidadores como: cansaço, distúrbio do sono, cefaleia, perda de peso, hipertensão e insatisfações na vida social (FELÍCIO et al., 2005), exclusão social, isolamento afetivo e social, depressão, erosão nos relacionamentos, perda da perspectiva de vida, distúrbios do sono, maior uso de psicotrópicos (FLORIANI, 2004).

Esses fatores associados podem restringir as possibilidades de o cuidador ter uma melhor qualidade de vida (BOCCHI, 2004). Além disso, a sobrecarga do cuidador pode reduzir a qualidade dos cuidados prestados e, consequentemente, pode afetar a saúde da pessoa que recebe cuidados (A'CAMPO; SPLIETHOFF-KAMMINGA, 2010).

$\mathrm{O}$ ato de cuidar não é uma tarefa de fácil execução, pois exige uma mudança radical na vida de quem cuida e também demanda a execução de tarefas complexas, delicadas e sofridas. Associado ao fato de que o cuidador geralmente é uma pessoa que se encontra em processo de envelhecimento, pode-se inferir que ele se torna um doente em potencial e sua capacidade funcional está constantemente em risco (LEAL, 2000; KARSCH, 2003).

Estudo realizado por Garrido e Menezes (2004) apontou que o cuidador que não recebe um suporte formal para atender às necessidades do indivíduo 
que precisa de cuidados corre o risco de, também, se tornar um paciente dentro do sistema. As tarefas que são atribuídas ao cuidador, geralmente sem receber orientação e suporte adequado, associada à alteração na rotina, e o tempo despendido no cuidado pode ter impacto negativo na qualidade de vida do cuidador (AMENDOLA; OLIVEIRA; ALVARENGA, 2008).

Para a Organização Mundial da Saúde qualidade de vida é a percepção do indivíduo sobre sua posição na vida no contexto da cultura e sistema de valores nos quais ele vive e em relação aos seus objetivos, expectativas, padrões e preocupações (WHOQOL GROUP, 1994). Para Minayo, Harz e Buss (2000) “a qualidade de vida é uma noção eminentemente humana, que tem sido aproximada ao grau de satisfação encontrada na vida familiar, amorosa, social e ambiental".

Uma revisão sistemática realizada por Kluthcovsky e Kluthcovsky (2009), sobre os estudos que utilizaram o WHOQOL-bref como instrumento para coleta de dados em avaliações de qualidade de vida encontrou apenas 11 estudos em que cuidadores foram os sujeitos estudados. Os mesmo autores ressaltam que, no Brasil, tem aumentado o interesse por estudos sobre os cuidados domiciliários de pessoas com perdas funcionais e dependência e sobre seus cuidadores. Apontam, também, a necessidade de políticas públicas efetivas, que ofereçam apoio às famílias de pessoas com perdas funcionais e dependência, ações fundamentais para a diminuição da sobrecarga do cuidador e a consequente melhora da qualidade de vida.

Bittencourt e Hoehne (2009) realizaram um estudo com familiares de crianças surdas e concluíram que apesar dos escores positivos obtidos para os domínios físico $(73,8)$ e relações sociais $(72,2)$, estes familiares carecem de maior suporte psicológico e do apoio de um sistema de redes sociais, para prover melhoria nas condições estruturais de vida.

A'Campo e Spliethoff-Kamminga (2010) sugerem que programas educacionais para cuidadores de pessoas com Parkinson poderiam contribuir para uma melhor qualidade de vida do parkinsoniano e do próprio cuidador.

Assim, estudar a qualidade de vida de cuidadores de pessoas com necessidades especiais pode contribuir para a elaboração de programas educacionais que favoreçam a qualidade de vida desses indivíduos, cuidadores e pessoas com necessidades especiais, bem como propor políticas públicas que favoreçam a melhora da saúde, bem como, a inclusão social de ambos.

Frente a essas considerações o presente estudo teve como objetivo analisar e comparar a qualidade de vida em saúde de cuidadores de pessoas com necessidades especiais em atendimento em uma instituição de reabilitação. Pretende-se identificar, comparar e correlacionar os domínios de qualidade de vida com três variáveis: idade da pessoa com necessidades especiais, idade do cuidador e tempo que presta cuidados. 


\section{Método}

\subsection{Participantes}

Foram participantes do estudo 90 cuidadores principais de pessoas com necessidades especiais em atendimento em uma instituição de reabilitação. Foram consideradas pessoas com necessidades especiais crianças e adultos com deficiência física, auditiva, intelectual e crianças com dificuldade de aprendizagem e na comunicação.

Adotaram-se os seguintes critérios para a inclusão de participantes no estudo: 1) prestar assistência ao indivíduo com necessidades especiais, a maior parte do dia; 2) não receber auxílio financeiro para exercer tais atividades; 3) auxiliar nas principais tarefas de cuidados; 4) exercer a tarefa de cuidador principal no mínimo há seis semanas; e 5) concordar em participar do estudo.

Os cuidadores foram convidados a participar do estudo e conheceram os objetivos e propósitos do mesmo, como também, ficaram cientes das etapas de desenvolvimento da pesquisa. As pessoas que aceitaram o convite assinaram o Termo de Consentimento Livre e Informado conforme determina a Resolução CNS-196/96.

A princípio foram coletados dados de 114 cuidadores, no entanto, foram descartados 14 questionários devido às características dos respondentes não corresponderem aos critérios de inclusão no estudo.

A viabilização do estudo ocorreu após o parecer favorável do projeto $\mathrm{n}^{\mathrm{O}}$ 547/2005 emitido pelo comitê de ética em pesquisa.

\subsection{Procedimentos PARA A COleta de dAdos}

Para a coleta de dados utilizaram-se dois instrumentos: 1) um questionário com perguntas estruturadas que versou sobre o perfil do cuidador e da pessoa com necessidades especiais em atendimento na instituição; 2) um questionário de medida de qualidade de vida, o WHOQOL-Bref, desenvolvido pela Organização Mundial de Saúde.

A construção do questionário estruturado que enfocou o perfil do cuidador apoiou-se em referenciais teóricos de estudos recentes sobre a temática. Após a elaboração do questionário este foi encaminhado para um juiz que verificou a pertinência e o conteúdo das perguntas, além da forma e do vocabulário utilizado. Em sua versão final, o questionário foi adequado conforme as sugestões fornecidas pelo juiz consultado.

Em relação ao questionário para medida de qualidade de vida optou-se pelo WHOQOL-Bref, por constituir-se um instrumento reconhecido internacionalmente e validado no Brasil. A versão em português do questionário de qualidade de vida WHOQOL-Bref foi considerada, após tradução e validação, como um instrumento com bom desempenho psicométrico aliado a uma facilidade de uso, tornando-se uma boa alternativa para avaliar qualidade vida no Brasil (FLECK et al.; 2000). 
O WHOQOL-bref é uma versão abreviada do WHOQOL-100, composto pelas 26 questões que obtiveram os melhores desempenhos psicométricos que resultaram em quatro domínios: físico, psicológico, relações sociais e meio ambiente (FLECK et al.; 2000). No domínio meio ambiente são abordadas questões relacionadas à segurança e proteção, ambiente no lar, recursos financeiros, cuidados de saúde, oportunidade de adquirir novas informações e habilidades, participação em atividades de lazer, trânsito e transporte. No domínio físico inclui questões sobre dor e desconforto, energia e fadiga, sono e repouso, mobilidade, atividades de vida diária, dependência de medicação ou tratamentos e capacidade para o trabalho. No domínio relações abrange questões sobre as relações pessoais, suporte social e atividade sexual. No domínio psicológico inclui questionamentos sobre os sentimentos positivos, pensar, aprender, memória, concentração, autoestima, imagem corporal, aparência, sentimentos negativos, espiritualidade, religião e crenças pessoais.

As respostas de todas as questões foram obtidas por meio de uma escala tipo Likert de cinco pontos, sendo que os escores mais altos denotaram melhor qualidade de vida.

A coleta de dados foi realizada de forma individual com os cuidadores durante o período em que estes aguardavam o final de atendimentos de seus familiares submetidos à terapias de reabilitação. $O$ contato inicial com os participantes ocorreu na sala de espera da instituição. Mediante o consentimento deles em participar da pesquisa foram encaminhados para uma sala preparada para a aplicação do questionário.

Para o preenchimento do questionário, o participante foi, previamente, orientado pela entrevistadora sobre o instrumento. Em alguns casos o participante solicitou ajuda para realizar o preenchimento do questionário. Nestes casos, era realizada a leitura da questão específica da dúvida e das alternativas de resposta e aguardava-se a manifestação do cuidador.

\subsection{Procedimentos PARA ANÁlise de dAdOS}

$\mathrm{Na}$ análise dos dados foram identificadas categorias referentes ao perfil do cuidador, visando à descrição e representação percentual da amostra estudada. Utilizou-se o teste não-paramétrico Kruskal-Wallis para verificar a ocorrência de diferença estatística significante na qualidade de vida de cinco grupos de cuidadores cuja classificação ocorreu dependendo dos déficits de seus familiares. O nível de significância adotado foi $\mathrm{p} \leq 0,05$.

\section{Resultados}

A análise dos dados possibilitou identificar cinco categorias referentes ao perfil do cuidador: 1) gênero; 2) vínculo com a pessoa com necessidade especial; 3) idade; 4) tempo em anos que exerce a atividade de cuidador; 5) estado de saúde. 
Em relação ao gênero verificou-se que 92\% (83/90) eram mulheres e apenas 8\% (7/90) eram homens.

Os dados do estudo também mostraram que os cuidadores principais eram: $79 \%$ as mães; $7 \%$ as avós; $6 \%$ os pais; $2 \%$ as tias; $2 \%$ as irmãs; $1 \%$ avô; $1 \%$ filha; $1 \%$ acompanhante; $1 \%$ filho.

Quanto à faixa etária verificou-se que a média de idade era 39,5 anos $( \pm 12$ anos), sendo que o cuidador mais velho tinha 71 anos e o mais novo 17 anos.

Os dados apontaram que 34 participantes eram cuidadores entre seis e 10 anos; 30 participantes entre um e cinco anos e 26 participantes eram cuidadores há mais de 11 anos.

Em relação ao estado de saúde: 49 participantes consideraram ter um bom estado de saúde; 21 muito bom; 13 nem bom e nem ruim; três fraco; três muito ruim e um não respondeu.

Em relação aos dados sobre qualidade de vida dos participantes do estudo verificou-se que o pior resultado encontrado foi no domínio meio ambiente 55,56\% $( \pm 15,23)$. Em contrapartida o domínio em que os participantes apontaram uma melhor qualidade de vida foi o físico $72,84 \%( \pm 15,21)$. O domínio relações sociais foi pontuado como segundo melhor 70,55\% $( \pm 16,51)$, e o domínio psicológico foi pontuado pelos cuidadores com $66,94 \%( \pm 15,34)$.

\subsection{Qualidade DE VIDA POR GRUPOS DE CUIDADORES}

Os participantes do estudo foram distribuídos em cinco grupos de cuidadores: 1) cuidadores de pessoas com dificuldades de aprendizagem; 2) cuidadores de pessoas com deficiência auditiva; 3) cuidadores de pessoas com dificuldade na comunicação; 4) cuidadores de pessoas com deficiência física; 5) cuidadores de pessoas com deficiência intelectual.

Na Tabela 1 são apresentados os dados referentes à média, ao desviopadrão e ao valor mínimo e máximo da qualidade de vida para cada grupo de cuidadores. De acordo com os dados apresentados, observou-se que os cuidadores de pessoas com dificuldade de aprendizagem apresentaram melhor qualidade de vida para o domínio físico $(66,67 \%)$ e pior qualidade de vida para o domínio meio ambiente $(48,92 \%)$. Os cuidadores de pessoas com deficiência auditiva tiveram melhor pontuação, em qualidade de vida, no domínio relações sociais $(77,78 \%)$ e a menor pontuação para o domínio meio ambiente $(60,42 \%)$. A maior pontuação encontrada ao analisar a qualidade de vida de cuidadores de pessoas com dificuldade na comunicação foi encontrada para o domínio físico $(75,54 \%)$ e a menor para o domínio meio ambiente $(53,79 \%)$. O maior percentual apontado para cuidadores de pessoas com deficiência física foi encontrado para o domínio físico $(72,98 \%)$ e o menor para o domínio meio ambiente $(58,02 \%)$. Os cuidadores de 
pessoas com deficiência intelectual tiveram maior pontuação para o domínio físico $(77,68 \%)$ e a menor pontuação $(63,44 \%)$ para o domínio meio ambiente.

Tabela 1 - Valores da média, desvio-padrão, mínimo e máximo da qualidade de vida para os diferentes grupos de cuidadores

\begin{tabular}{lllll}
\hline \multicolumn{1}{c}{$\%$} & \multicolumn{1}{c}{$\begin{array}{c}\text { Físico } \\
\text { Média }( \pm \mathrm{DP}) \\
\text { Min-Max }\end{array}$} & $\begin{array}{c}\text { Psicológico } \\
\text { Média }( \pm \mathrm{DP}) \\
\text { Min-Max }\end{array}$ & $\begin{array}{c}\text { Relações sociais } \\
\text { Média }( \pm \mathrm{DP}) \\
\text { Min-Max }\end{array}$ & $\begin{array}{c}\text { Meio ambiente } \\
\text { Média }( \pm \mathrm{DP}) \\
\text { Min-Max }\end{array}$ \\
\hline $\begin{array}{l}\text { Dificuldade de } \\
\text { aprendizagem }\end{array}$ & $\begin{array}{c}66,67( \pm 19,40) \\
\text { 35,7-85,7 }\end{array}$ & $\begin{array}{l}65,97( \pm 13,39) \\
41,7-83,3\end{array}$ & $\begin{array}{l}63,19( \pm 15,67) 41,7 \\
-91,7\end{array}$ & $\begin{array}{c}48,92( \pm 12,02) 28,1 \\
-71,9\end{array}$ \\
$\begin{array}{l}\text { Deficiência } \\
\text { auditiva }\end{array}$ & $70,24( \pm 11,44)$ & $67,36( \pm 6,13) 62,5$ & $77,78( \pm 8,61) 66,7$ & $60,42( \pm 14,34) 46,9$ \\
Dificuldade na & $75,6-85,7$ & $-75,0$ & $-91,7$ & $-84,4$ \\
comunicação & $35,7-100,0$ & $20,8-91,7$ & $-100,0$ & $53,79( \pm 17,04) 12,5$ \\
Deficiência & $72,98( \pm 17,88)$ & $64,67( \pm 17,63)$ & $69,02( \pm 15,93) 37,5$ & $58,02( \pm 15,79) 18,8$ \\
física & $28,6-100,0$ & $29,2-91,7$ & $-100,0$ & $-93,8$ \\
Deficiência & $77,68( \pm 8,72)$ & $70,42( \pm 10,48)$ & $74,17( \pm 9,17) 58,3$ & $63,44( \pm 8,60) 46,9-$ \\
intelectual & $62,5-89,3$ & $58,3-91,7$ & $-91,7$ & 75,0 \\
\hline
\end{tabular}

A análise estatística por meio do teste Kruskal Wallis demonstrou que não havia diferença significativa entre os cuidadores dos diferentes grupos de pessoas com necessidades especiais para o domínio físico $(\mathrm{p}=0,6991)$; psicológico $(p=0,6885)$; relações sociais $(p=0,1730)$ e meio ambiente $(p=0.1240)$.

\subsection{CORRELAÇ̃̃o QUALIDADE DE VIDA E IDADE DO CUIDADOR}

Os resultados indicaram que a idade do cuidador não influenciou em sua qualidade de vida em relação aos domínios psicológico, físico, meio ambiente e relações sociais (Tabela 2 ).

Tabela 2 - Coeficiente de correlação entre idade do cuidador e escores obtidos nos diferentes domínios da WHOQOL-Bref conforme determinado pelo coeficiente de correlação de Spearman

\begin{tabular}{llc}
\hline & & Coeficiente de correlação (r) \\
\hline Correlação entre idade do cuidador e & Psicológico & $-0,053$ \\
os domínios de qualidade de vida & Meio ambiente & $-0,125$ \\
& Físico & $-0,149$ \\
& Relações sociais & 0,075 \\
\hline
\end{tabular}




\subsection{CoRRElAÇÃo QUALIDADE DE VIDA E IDADE DA PESSOA COM NECESSIDADE ESPECIAL}

A idade da pessoa que recebe os cuidados não influenciou na qualidade de vida dos participantes do estudo para o domínio psicológico, meio ambiente e relações sociais. No entanto, a correlação foi considerada significante para o domínio físico (Tabela 3). Esse resultado mostrou que quanto mais velha a pessoa com necessidade especial, pior a qualidade de vida do cuidador no domínio físico.

Tabela 3 - Coeficiente de correlação entre idade da pessoa com necessidade especial e escores obtidos nos diferentes domínios da WHOQOL-Bref conforme determinado pelo coeficiente de correlação de Spearman

\begin{tabular}{lll}
\hline & & Coeficiente de correlação $(\mathrm{r})$ \\
\hline $\begin{array}{lll}\text { Correlação entre idade da pessoa } \\
\text { com necessidade especial e os }\end{array}$ & Meio ambiente & $-0,091$ \\
domínios de qualidade de vida & Físico & $-0,125$ \\
& Relações sociais & 0,074 \\
\hline
\end{tabular}

${ }^{*} \mathrm{p}=0,047$

\subsection{Correlação QUALIDAde DE VIDA E TEMPO DE CUIDADO}

Na Tabela 4 são apresentados os dados de correlação entre qualidade de vida do cuidador nos diferentes domínios e o tempo que ele presta cuidados à pessoa com necessidade especial. Os dados indicaram que a qualidade de vida dos participantes não foi influenciada pelo tempo que o indivíduo exerce a atividade.

Tabela 4 - Coeficiente de correlação entre o tempo que presta cuidado para a pessoa com necessidade especial e escores obtidos nos diferentes domínios da WHOQOLBref conforme determinado pelo coeficiente de correlação de Spearman

\begin{tabular}{lll}
\hline & & Coeficiente de correlação (r) \\
\hline Correlação entre tempo que presta & Psicológico & 0,046 \\
$\begin{array}{l}\text { cuidado para pessoa com necessidade } \\
\text { especial e os domínios de qualidade de }\end{array}$ & Físico & $-0,047$ \\
vida & Relações sociais & 0,156 \\
\hline
\end{tabular}

\section{Discussão}

Este estudo mostrou uma prevalência de cuidadores do gênero feminino. Gonçalves et al. (2006), Makiyama et al. (2004) e Amendola , Oliveira , Alvarenga (2008) ao estudar cuidadores de pacientes adultos, encontraram que essa atividade era realizada por mulheres. Isto parece indicar que, independente da faixa etária 
de quem recebe os cuidados e do tipo da patologia que apresentam, geralmente é a mulher que assume a função de cuidadora principal.

Quanto ao grau de parentesco, neste estudo, as mães eram as responsáveis pela assistência. Para Borges (2006) "Quem mais assume o papel de cuidador são as mulheres: a esposa, a filha, a irmã, a sobrinha, a cuidadora profissional, a enfermeira...". Por outro lado, no estudo de Felício et al. (2005) os filhos constituíram a maioria dos cuidadores principais. Observa-se, no entanto, que os estudos destes pesquisadores foram realizados com cuidadores de pessoas idosas e adultas. Estudo realizado por Vidal, Duffau e Ubilla (2007) encontrou que crianças asmáticas eram cuidadas pelas mães. Assim, o grau de parentesco do cuidador pode ter relação com a idade da pessoa que está sob seus cuidados.

Segundo Karsch (2003), o fator predominantemente cultural pode ser o determinante para que, no Brasil, ainda, hoje, o papel de cuidador seja de responsabilidade da mulher.

Em relação ao estado civil os resultados deste estudo coincidem com os encontrados por Roig, Abengózar e Serra (1998), Kluthcovsky e Takayanagui (2007) e Gonçalves et al (2006), demonstrando a prevalência de cuidadores casados.

Quanto à atividade profissional exercida pelo cuidador, grande parte dos participantes refere que exerce a função de cuidador 24 horas por dia. Relato semelhante foi encontrado no estudo realizado por Makiyama et al. (2004). Estes dados coincidem, também, com os de Braccialli, Reganhan e Manzini (2005) e com os de Gonçalves et al. (2006). Conforme esses autores, o cuidador não exerce atividade profissional formal, e essa seria uma forma das famílias se adequarem para atender as necessidades de cuidados desta população.

Esses dados também estão em consonância com os encontrados no estudo de Gonçalves (2002), no qual 57,9\% dos participantes relataram que prestavam cuidados entre 18 e 24 horas diária. De acordo com a mesma autora o fato de o cuidador prover cuidados por 24 horas ao dia ao individuo dependente pode causar estresse social ao cuidador por levar ao afastamento, muitas vezes, da sua própria família, dos amigos e da vida social. Gonçalves et al. (2006) também confirmaram em seu estudo que a maioria dos cuidadores se dedica de modo permanente, estando expostos a constantes riscos de adoecimento e sobrecarga. Tal sobrecarga compromete o autocuidado, os cuidadores relatam não ter mais tempo para cuidar de si próprios.

Os dados encontrados nesta presente investigação indicaram que a maioria dos cuidadores dos pacientes da instituição pesquisada exerce a função de cuidador principal há muito tempo. Para Gonçalves (2002) o número de anos na função e o tempo diário despendido com cuidados podem refletir em sobrecarga física, deixando os cuidadores suscetíveis a diferentes doenças.

Neste estudo a maioria dos cuidadores relatou não apresentar nenhum problema de saúde e considerou seu estado de saúde como bom. Estudos realizados 
por Gonçalves (2002) e Karsch (2003) apresentaram resultados diferentes. Esses autores encontraram, respectivamente, que 63,2\% dos cuidadores relataram ter alguma patologia diagnosticada por médico, e 100\% dos cuidadores relataram problemas de saúde.

Gonçalves et al. (2006) questionaram sobre a situação de saúde de cuidadores de idosos, doenças ou problemas que os afetavam, e, nessa investigação, os cuidadores referiram sofrer de hipertensão arterial e outros problemas cardiovasculares, seguido de problemas osteomusculares e diabetes mellitus. Contudo, a maioria considerou seu estado de saúde como bom. E quando questionados sobre sua qualidade de vida, a maioria dos participantes do estudo mostrou-se satisfeita. Kluthcovsky e Takayanagui (2007) também relataram em sua pesquisa sobre a autopercepção que os agentes comunitários de saúde têm sobre sua saúde, tendo a maioria a avaliado como boa.

De acordo com os dados obtidos na presente pesquisa, em relação aos domínios de qualidade de vida pesquisados, o meio ambiente obteve a menor pontuação. Esses dados coincidem com os encontrados por Gonçalves et al. (2006), que concluíram que entre as consequências da tarefa de cuidar do idoso, as cuidadoras tinham limitações na vida profissional, desde a redução da jornada de trabalho até o seu abandono e expressavam falta de tempo para se cuidar, convivência conjugal com conflitos, cansaço permanente e percepção de saúde piorada, sendo essas relacionadas ao domínio meio ambiente.

O domínio que obteve melhor pontuação dos participantes do estudo foi o físico. Resultado semelhante foi encontrado por Kluthcovsky e Takayanagui (2007) que estudaram a qualidade de vida de cuidadores formais, agentes comunitários de saúde e por Amendola, Oliveira e Alvarenga (2008) que estudaram qualidade de vida de cuidadores de pessoas dependentes funcionalmente.

Quando se discute qualidade de vida deve-se considerar a percepção do indivíduo. Paschoal (2001 apud KLUTHCOVSKY; TAKAYANAGUI, 2007, p. 182) enfatizou a importância da avaliação da qualidade de vida percebida pela pessoa, valorizando assim, a opinião do indivíduo. A qualidade de vida teria um conceito diferente de pessoa para pessoa, tendendo a mudar ao longo da vida. Para Arnold et al. (2004 apud KLUTHCOVSKY; TAKAYANAGUI, 2007, p. 182) o julgamento que cada indivíduo faz de sua qualidade de vida global deve ser considerado. Espera-se que o indivíduo combine diferentes aspectos de sua vida e os resuma em uma avaliação global de sua qualidade de vida.

A maioria das correlações apresentadas não foi considerada significante. Houve significância, apenas, entre a qualidade de vida do cuidador no domínio físico e a idade da pessoa com necessidade especial, tendo-se encontrado uma correlação negativa entre essas variáveis. Esse resultado corrobora com os achados de Amendola, Oliveira e Alvarenga (2008) que consideram que indivíduos com limitações funcionais mais velhos requerem cuidados que sobrecarregam fisicamente o cuidador. 


\section{Conclusão}

O perfil dos cuidadores dos pacientes da instituição estudada não difere daquele encontrado em outros estudos, geralmente são mulheres, exercem esta função há vários anos e despendem grande parte de horas diárias nessa atividade.

A qualidade de vida de acordo com os cuidadores foi classificada como boa e o domínio ambiental recebeu a menor pontuação, resultados estes que coincidem com os obtidos em outros estudos. É importante ressaltar que dentre as correlações estudadas, a única que se apresentou significativa foi à relação entre domínio físico e idade da pessoa com necessidades especiais, que também não difere de resultados de outros estudos, indicando que quanto mais velha a pessoa com necessidades especiais, mais difícil e penoso é o ato de cuidar.

Os resultados obtidos neste estudo indicam a necessidade de desenvolvimento de programas de educação informal com ações na interface entre as áreas da saúde e educação, visando à prevenção de malefícios à qualidade de vida do cuidador familiar.

No âmbito educacional poderiam ser propostos programas de orientação a cuidadores que abrangessem aspectos relacionados ao domínio meio ambiente, uma vez que diferentes estudos com cuidadores de pessoas com diferentes necessidades têm apontado uma fragilidade na qualidade de vida quanto a esse aspecto. O programa educacional poderia abordar questões de interesse para a área de Educação Especial, tais como: (a) adequação do ambiente domiciliar às necessidades de cada individuo; (b) informações sobre os programas governamentais existentes no país para obtenção de auxílios financeiros para a pessoa com necessidades especiais; (c) cuidados com a saúde do cuidador e da pessoa dependente; (d) orientações sobre as habilidades e dificuldades da pessoa com necessidades especiais, bem como os mecanismos e programas existentes na comunidade que ofereçam oportunidade de adquirir novas informações e habilidades; (e) orientações em relação à participação em atividades de lazer, e a disponibilidade de transporte público adequado às necessidades do usuário; (f) orientações sobre os direitos da pessoa com necessidades especiais.

No âmbito da saúde, deve-se considerar que os avanços dos últimos anos, seja na medicina ou em outras áreas como fisioterapia, terapia ocupacional, fonoaudiologia, psicologia e nutrição, têm proporcionado, mais especificamente ao indivíduo com deficiência, o aumento em sua expectativa de vida. Com o aumento da expectativa de vida da pessoa com deficiência, há necessidade de ações públicas para prover as necessidades desses indivíduos, a fim de que os mesmos tenham qualidade vida adequada e cuidados necessários. Os dados, desse e de outros estudos realizados, indicam que a idade avançada do cuidador associada ao aumento de idade da pessoa que recebe cuidados tem trazido prejuízos, tanto para sua qualidade de vida quanto para a qualidade de vida da pessoa dependente. 


\section{REFERÊNCIAS}

A'CAMPO, L. E. I; SPLIETHOFF-KAMMINGA, N. G. A. Caregiver education in Parkinson's disease: formative evaluation of a standardized program in seven European countries. Qual Life Res, Oxford, v.19, p.55-64, 2010.

AMENDOLA, F.; OLIVEIRA, M.A.C.; ALVARENGA, M.R.M. Qualidade de vida dos cuidadores de pacientes dependentes no programa de saúde da família. Texto Contexto Enferm, Florianópolis, v.17, n.2, p.266-272, 2008.

AMERICAN OCCUPATIONAL THERAPY ASSOCIATION. Estrutura da prática da terapia ocupacional: domínio e processo. 2. ed. Tradução Daniel Gustavo de Sousa Carleto et al. Rev. Triang.: Ens. Pesq. Ext., Uberaba, v.3, n 2, p 57-147, 2010.

BITTENCOURT, Z. Z. L. C.; HOEHNE, E. L. Qualidade de vida de familiares de pessoas surdas atendidas em um centro de reabilitação. Ciênc. saúde coletiva, Rio de Janeiro, v.14, n.4, 2009 .

BOCCHI, S.C.M. Vivenciando a sobrecarga ao vir-a-ser um cuidador familiar de pessoa com acidente vascular cerebral (avc): uma análise do conhecimento. Rev. Latino- Am Enfermagem, Ribeirão Preto, v.12, n.1, p.115-21, 2004.

BORGES MF. Manual do cuidador. Disponível em: <http:/ / www.alzheimer.med.br>. Acesso em: 12 jan. 2006.

BOVER, A. Cuidadores informales de salud del ámbito domiciliario: percepciones y estrategias de cuidado ligadas al género y a la generación. 2004. 761f. Tese (Doutorado em Ciências da Educação) - Departament de Ciències de l'Educació, Universitat de Les Illes Balears, Palma de Mallorca. 2004.

BRACCIALLI, L.M.P; REGANHAN, W.G; MANZINI, E.J. Lazer, escola e ocupação do tempo livre: análise de relatos de alunos com paralisia cerebral. Arq Bras Paralisia Cerebral, São Paulo, v.2, n.4, p.26-35, 2005.

DWYER, J.W; LEE, G.R.Y; JANKOWSKI, T.B. Reciprocity, elder satisfaction, and caregiver stress and burden: the exchange of aid in the family caregiving relationship. $J$ Marriage Fam, Oxford, v.56, n.1, p.35-43, 1994.

FELÍCIO, D.N.L. et al. Atuação do fisioterapeuta no atendimento domiciliar de pacientes neurológicos: a efetividade sob a visão do cuidador. Rev Bras Promoção Saúde, Fortaleza, v.18, n.2, p.64-69, 2005.

FLECK, M.P.A. et al. Aplicação da versão em português do instrumento abreviado de avaliação da qualidade de vida "WHOQOL-bref". Rev. Saúde Pública, São Paulo, v.34, n.2, 2000.

FLORIANI, C.A. Cuidador familiar: sobrecarga e proteção. Secretaria de Saúde de Estado do Rio de Janeiro. Rev Bras Cancerologia, Rio de Janeiro, v.50, n.4, p.341-345, 2004.

GARRIDO, R.; ALMEIDA, O.P. Distúrbios de comportamento em pacientes com demência: impacto sobre a vida do cuidador. Arq Neuropsiquiatr, São Paulo, v.57, n.2-B, p.427-434, 1999.

GARRIDO, R.; MENEZES, P.R. Impacto em cuidadores de idosos com demência atendidos em um serviço psicogeriátrico. Rev Saúde Pública, São Paulo, v.38, n.6, p.835-1, 2004. 
GONÇALVES, L.H.T. et al. Perfil da família cuidadora de idoso doente/fragilizado do contexto sociocultural de Florianópolis, SC. Texto E Contexto Enferm, Florianópolis, v.15, n.4, p.570-7, 2006.

GONÇALVES, L.O. Cuidadores primários familiares dos idosos atendidos na Clínica Escola de Fisioterapia da Universidade do Vale do Itajaí. 2002. 91f. Dissertação (Mestrado em Engenharia de produção) - Universidade Federal de Santa Catarina, Florianópolis. 2002.

HINRICHSEN, G.A.; NIEDEREHE, G. Dementia management strategies and adjustment of family members of older patients. Gerontologist, Washington, v. 34, p.95-102, 1994.

KARSCH, U.M. Idosos dependentes: famílias e cuidadores. Cad Saúde Pública, Rio de Janeiro, v.19, n.3, p.861-866, 2003.

KLUTHCOVSKY, A.C.G.C; TAKAYANAGUI, A.M.M. Qualidade de vida - aspectos conceituais. Rev Salus-PR, Guarapuava, v.1, n.1, p.13-15, 2007.

KLUTHCOVSKY, A. C. G.C.; KLUTHCOVSKY, F. A. O WHOQOL-bref, um instrumento para avaliar qualidade de vida: uma revisão sistemática. Rev. psiquiatr. Rio Gd. Sul, Porto Alegre, v.31, n.3, 2009.

LEAL, M.G.S. O desafio da longevidade e o suporte ao cuidador. Rev Terceira Idade, São Paulo, v.11, n.20, p. 19-29, 2000.

MAKIYAMA, T.Y. et al. Estudo sobre a qualidade de vida de pacienteshemiplégicos por acidente vascular cerebral e de seus cuidadores. Acta Fisiatr, São Paulo, v.11, n.3, p.106109, 2004.

MENDES, P.B.M.T. Cuidadores: heróis anônimos do cotidiano. 1995. 195f. Dissertação (Mestrado em Serviço Social) - Pontifícia Universidade Católica - PUC, São Paulo. 1995.

MINAYO, M. C. S.; HARZ, Z. M. A.; BUSS, P. M. Qualidade de vida e saúde: um debate necessário. Ciência Saúde Coletiva, Rio de Janeiro, v.5, p.7-18, 2000.

ROIG, M.V; ABENGÓZAR, M.C; SERRA, E. La sobrecarga en los cuidadores principales de enfermos de Alzheimer. Anal Psicologia, Murcia, v.14, n.2, p.215-227, 1998.

VIDAL, A.G; DUFFAU, G.T; UBILLA, C.P. Calidad de vida en el niño asmático y su cuidador. Rev Chil Enf Respir, Santiago, v.23, p.160-166, 2007.

WESTPHAL, A. et al. Comparação da qualidade de vida e sobrecarga dos cuidadores de pacientes com epilepsia por esclerose mesial temporal e epilepsia mioclônica juvenil. J Epilepsy Clin Neurophysiol, Porto Alegre, v.11, n.2, p.71-76, 2005.

WILSON, H.S. Family caregiving for a relative with Alzheimer's Dementia. Nurs Res, New york, v. 38, n.2, p.94-98, 1989.

WHOQOL Group. The development of the World Health Organization quality of life assessment instrument (the WHOQOL). In: ORLEY, J; KUYKEN, W. Quality of life assessment: international perspectives. Heidelberg: Springer Verlag, 1994. p.41-60,

Recebido em: 19/04/2011

Reformulado em: 06/09/2011

Aprovado em 14/10/2011 